\title{
PLANEJAMENTO EXPERIMENTAL APLICADO À SEDIMENTAÇÃO DE LAMAS FOSFÁTICAS
}

\author{
P. C. ANDRADE ${ }^{1}$, G. E. S. VALADÃO ${ }^{2}$ \\ ${ }^{1}$ Instituto Federal do Sertão Pernambucano - Campus Ouricuri., ${ }^{2}$ Universidade Federal de Minas Gerais \\ Departamento de Engenharia de Minas \\ patricia.andrade@ifsertao-pe.edu.br
}

Submetido 14/11/2017 - Aceito 28/11/2017

DOI: $10.15628 /$ holos.2017.6543

\section{RESUMO}

A lama gerada no processamento de minérios contém grande quantidade de partículas finas, estáveis em suspensões aquosas, e que apresentam velocidade de sedimentação extremamente baixa. Normalmente reagentes coagulantes e floculantes são utilizados para agregar estas partículas para que se sedimentem com maior velocidade. O objetivo do trabalho é realizar testes de sedimentação em laboratório utilizando planejamento experimental. A amostra mineral utilizada é uma lama obtida a partir do processamento de minério de fosfato. Os testes de sedimentação foram realizados com 10\% de sólido em massa em proveta de $2000 \mathrm{~mL}$. Reagentes floculantes comerciais foram testados. O algoritmo de Yates foi utilizado para definir três variáveis: $\mathrm{pH}$, dosagem de coagulante e dosagem de floculante, em níveis superiores e inferiores para cada variável. Estes valores foram otimizados pelo Método do Passo Ascendente definindo assim a condição ideal para cada tipo de floculante. Obteve-se uma expressão matemática para cada tipo de floculante e, através do teste de Fisher, verificou-se a validade para cada equação matemática obtida. Os resultados mostraram que as variáveis e suas interações são significativas.

PALAVRAS-CHAVE: floculante, coagulante, método do passo ascendente, algoritmo de Yates, teste de Fisher

\section{EXPERIMENTAL PLANNING APPLIED TO SEDIMENTATION OF PHOSPHIC SLIMES}

\begin{abstract}
The slime obtained from ore processing contains large amounts of fine particles, stable in aqueous suspensions, and presenting extremely slow settling velocity. Normally, coagulant and flocculant reagents are used to aggregate these particles so that they can settle more rapidly. The objective of this work is to perform sedimentation tests at laboratory using an experimental design. The mineral sample utilized is a slime obtained from phosphate ore processing. The settling tests were carried out considering $10 \%$ of solids in mass, and using graduated cylinder of $2000 \mathrm{~mL}$. Commercial flocculant
\end{abstract}

reagents were tested. The Yates algorithm was utilized for definition of three variable: $\mathrm{pH}$, coagulant dosage and flocculant dosage, at higher and lower levels for each variable. These values were then optimized by the StepUp Method, thus defining an ideal condition for each type of flocculant. A mathematical expression was obtained for each type of flocculant and, from the Fisher's test, the validity of equations were verified. The results showed that variables and their interactions are significant.

KEYWORDS: flocculant, coagulant, ascending step method, Yates algorithm, Fisher test. 


\section{INTRODUÇÃO}

A lama gerada no processamento de minérios contém grande quantidade de partículas finas, estáveis em suspensões aquosas, e que apresentam velocidade de sedimentação extremamente baixa.

Normalmente reagentes coagulantes e floculantes são utilizados para agregar estas partículas para que se sedimentem com maior velocidade obtendo-se também líquidos sobrenadantes mais clarificados.

Planejamentos experimentais, baseados em princípios estatísticos, permitem extrair importantes informações de um sistema em estudo, realizando-se um número pequeno de experimentos. Os métodos utilizados permitem resolver problemas e tendo potencial de baixar drasticamente custo e reduzir tempo de desenvolvimento de novos produtos ou processos (Montgomery e Runger, 2003). Podem ser aplicados para: identificação de problemas do processo; desenvolvimento e otimização de processos; avaliação do material e alternativas; confiabilidade e teste de durabilidade; teste de desempenho; configuração de projeto do produto; determinação da tolerância do componente.

Segundo Duckworth (1960), o planejamento fatorial nos permite variar condições experimentais simultaneamente, mas de uma forma controlada e planejada. 0 método consiste na adoção de valores, níveis, superiores e inferiores para os parâmetros adotados. Assim, parâmetros e níveis experimentais podem ser combinados entre si e o efeito da sinergia das variáveis pode ser avaliado.

\section{REVISÃO BIBLIOGRÁFICA}

\subsection{Mecanismos de agregação de partículas}

\subsubsection{Coagulação}

A coagulação é obtida com a redução ou mesmo a eliminação da barreira energética repulsiva. A Figura 1 representa a compressão da dupla camada elétrica (KIM, 1995).

Para minimizar a barreira energética repulsiva é necessário:alterar a carga elétrica superficial da partícula por ação de íons determinantes de potencial (idp): controlando o $\mathrm{pH}$ ;promover adsorção específica de íons: a presença de íons especificamente adsorvidos provoca o abaixamento do potencial zeta, se forem cargas opostas à da superfície;provoca a compressão da dupla camada elétrica: adicionando eletrólitos.Usam-se em geral cátions hidrolisáveis como $\mathrm{Al}^{+} \mathrm{e}$ $\mathrm{Fe}^{+}$e alguns polímeros sintéticos com baixo peso molecular $\left(2 \times 10^{4}\right.$ a $\left.1 \times 10^{5}\right)$ geralmente catiônicos, (RICHSTFDON E CONNELY, 1988; HOGG , 2000). 


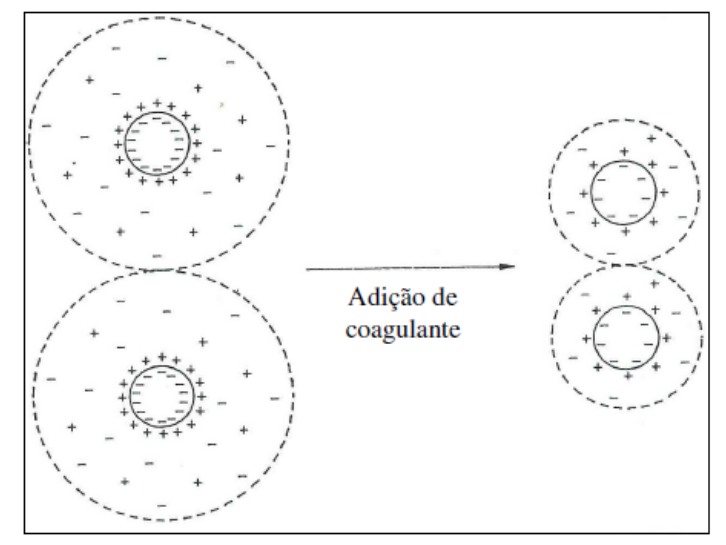

Figura 1: Compressão da dupla camada elétrica (KIM, 1995)

A regra de Schulze-Hardy afirma que a concentração de eletrólito necessária para induzir a coagulação em um sistema estabilizado de partículas carregadas é inversamente proporcional à valência do contra-íon (HUNTER, 1994). A natureza do contra-íon, a carga do co-íon e a natureza e concentração do solvente exercem pouca influência. Com relação ao primeiro fator, tem-se, geralmente, que quanto maior o contra-íon, menor o raio de hidratação e, logo, o contra-íon pode se aproximar mais da superfície da partícula. Desta maneira, contra-íons maiores possuem um efeito desestabilizador maior do que contra-íons menores, pois estes possuem maior raio de hidratação (VERRAL, WARWICK E FAIRHURST, 1999).

\subsubsection{Floculação}

A floculação é um processo de agregação caracterizado pela ligação entre as partículas que anteriormente estavam dispersas, pelo estabelecimento de pontes. Estas pontes são polímeros de alto peso molecular e filamentoso (LA MER e HEALY, 1963), que pode ser vista na Figura 2. Este processo pode ser descrito em duas etapas principais: adsorção do polímero na superfície das partículas e agregação das partículas formando flocos.

A adsorção em um substrato é normalmente atribuída a interações eletrostáticas ou ligações de hidrogênio. Polieletrólitos catiônicos e aniônicos adsorvem em partículas de carga oposta resultando em neutralização de cargas enquanto polímeros não iônicos são adsorvidos por ligações de hidrogênio com os grupos hidroxila do substrato.

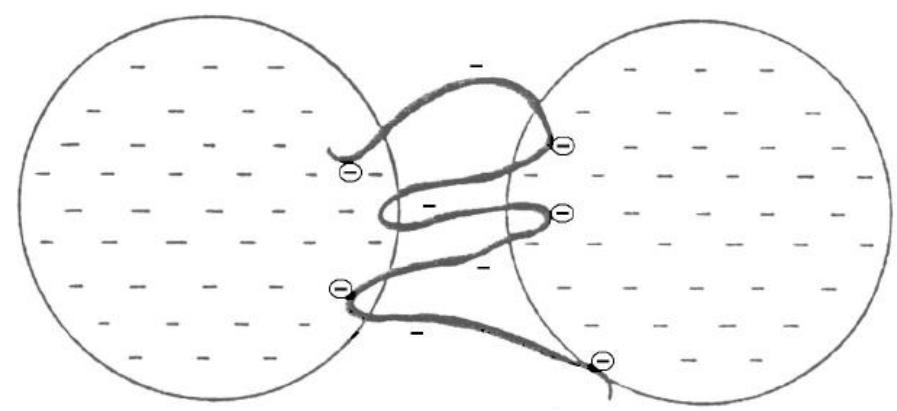

Figura 2: Floculação por pontes: polímero filamentoso de massa molar elevada (LA MER E HEALY, 1963) 
A floculação é um processo dinâmico e envolve adsorção de polímero e colisão entre partículas que resultam na formação e crescimento dos flocos. KISSA (1999) considera este processo reversível uma vez que há um equilíbrio entre crescimento e quebra dos flocos formados, por exemplo, por agitação vigorosa. SWIFT et al (2004) descobriram que o tamanho dos flocos aumenta rapidamente até um valor máximo e, então, diminui em uma taxa mais baixa do que a taxa inicial de agregação.

Segundo os trabalhos de SHARMA et al. (2006), a floculação é um processo que tem como finalidade formar agregados de partículas finamente divididas através da formação de flóculos que melhoram a sedimentação e causam a clarificação do sistema. A Figura 3 representa este processo de crescimento rápido do floco. Apesar da água retida diminuir a densidade média, os flocos apresentam alta velocidade de sedimentação, devido ao seu tamanho. A reconstrução dos flocos destruídos pelas tensões de cisalhamento é mais difícil nos sistemas onde predomina este tipo de mecanismo (CLAESSON et al.,1994) devido à quebra de moléculas do polímero.

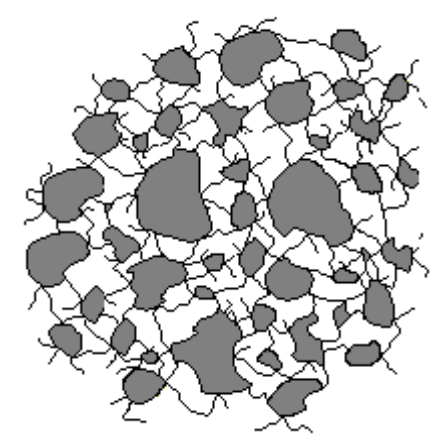

Figura 3 : Estrutura dos flocos resultante do mecanismo de adsorção do floculante (floco aberto) (BALTAR, 2004)

Em altas concentrações, é possível que o polímero se adsorva completamente em partículas individuais, antes que ocorra a formação de pontes, e que a repulsão entre as cargas do polímero cause a dispersão das partículas, como pode ser visto na Figura 4. Deste modo, polímeros de baixo peso molecular normalmente atuam como dispersantes. Por outro lado, a dosagem excessiva de polímero pode levar a um processo de superfloculação em que grandes flocos independentes são formados (KIRWAN, 2002).

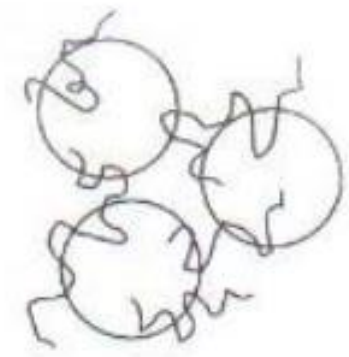

(A)

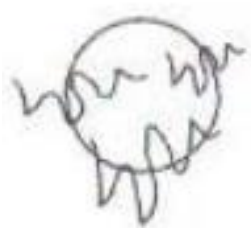

(B)

Figura 4 : Figura (A) Polímero promovendo a ligação entre partículas (agregação), (B) re-estabilização de partículas coloidais (rompimento do floco) (SHARMA et.al,2006) 
KISSA (1999) considera este processo reversível uma vez que há um equilíbrio entre crescimento e quebra dos flocos formados, por exemplo, por agitação vigorosa. SWIFT et al (2004) descobriram que o tamanho dos flocos aumenta rapidamente até um valor máximo e, então, diminui em uma taxa mais baixa do que a taxa inicial de agregação.

\subsection{Sedimentação}

A operação de sedimentação se baseia no fenômeno de transporte da partícula para o fundo de um equipamento, onde a partícula sólida em suspensão sofre ação das forças: gravidade, empuxo e resistência ao movimento (BENVINDO et al. 2002).

Segundo CHAVES (2004), se uma amostra previamente homogeneizada da suspensão for colocada em um tubo de vidro graduada de seção constante e deixada em repouso verifica-se que, certo tempo, as partículas mais grossas depositam no fundo do tubo e as partículas mais finas continuam em suspensão. As partículas intermediárias ficam distribuídas em diversas alturas de acordo com sua granulometria.

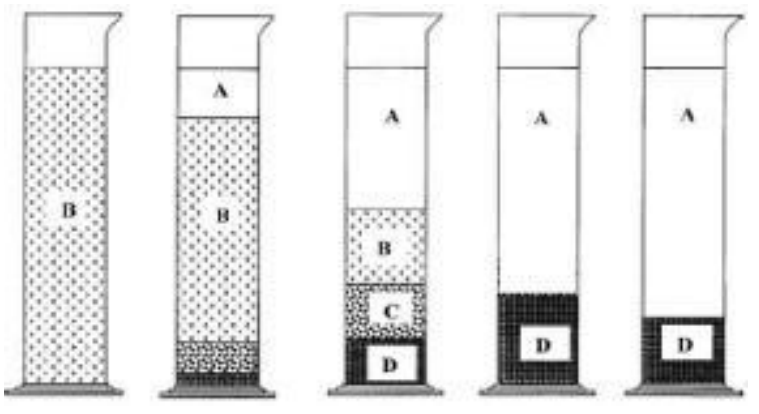

Figura 5: Etapas de teste de proveta e regiões formadas durante a sedimentação (FAUST, 1982).

Na Figura 5 , podem-se identificar quatro zonas distintas:

A - Líquido clarificado; B - Suspensão com concentração uniforme, igual à inicial; C - Zona de transição e concentração não uniforme, aumentando de cima para baixo; D - Zona de compressão, que aumenta com o passar do tempo.

No início do teste, a suspensão apresenta-se homogênea e a concentração de sólidos é constante em todos os pontos da proveta. Em seguida, as partículas maiores começam a sedimentar formando uma fina camada de sólidos no fundo da proveta, na região de compactação. Assim, esta região é formada por partículas sólidas mais pesadas e com maior velocidade de sedimentação. A sedimentação das partículas mais finas ocorre mais lentamente, sem interação entre elas, em uma região intermediária, de sedimentação livre, com concentração de sólidos constante. Na medida em que os sólidos começam a sedimentar, inicia-se a formação de uma região de líquido clarificado, isenta de sólidos.

No decorrer do teste, são observadas variações na altura das regiões. As regiões de líquido clarificado e de compactação tornam-se maiores devido ao desaparecimento da região de sedimentação livre. Em determinado momento, na fase final do teste, passam a existir apenas a 
região de compactação e a região de líquido clarificado. A partir desse ponto, o processo de sedimentação consiste em uma compressão lenta dos sólidos, que expulsa o líquido existente entre as partículas para a região de líquido clarificado. A expulsão do líquido promove a acomodação das partículas sólidas, que pode ser observado por meio de uma pequena variação na altura da região de compactação (LUZ et al, 2004).

A partir dos dados de altura da interface obtidos em função do tempo de teste, são traçadas curvas de sedimentação, como esquematizado na Figura 6, para obtenção de parâmetros como velocidade inicial de sedimentação, altura do ponto de compressão, etc.

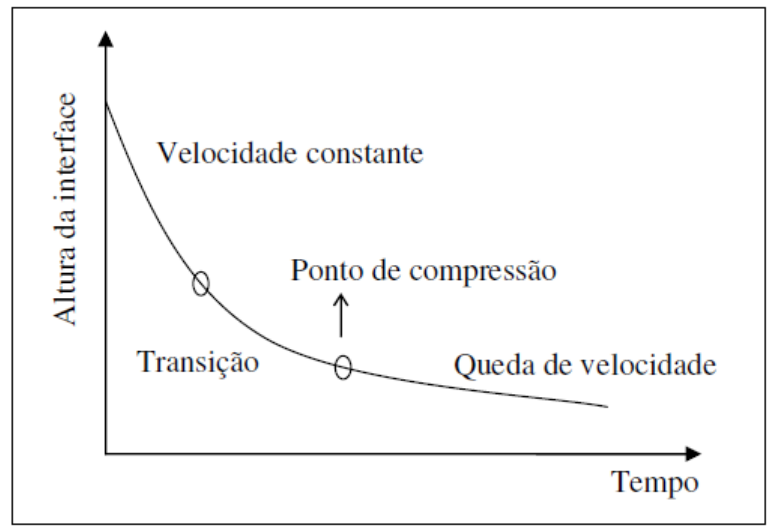

Figura 6: Curva de sedimentação.

As curvas de sedimentação apresentam, em geral, três seções: seção de velocidade constante, seção de transição e seção de queda de velocidade (compressão). Para determinação da velocidade de sedimentação, através do cálculo da inclinação da curva, considera-se somente a primeira seção, onde esta é constante (VALADÃO, 2011).

\subsection{Planejamentos experimentais}

Planejamentos experimentais baseados em princípios estatísticos permitem extrair do sistema em estudo o máximo de informação útil, fazendo um número mínimo de experimentos. Nesses experimentos, todos os fatores variam ao mesmo tempo, ou seja, todas as combinações possíveis dos níveis dos fatores são investigadas, sendo possível observar a interação entre os fatores.

Por meio da análise do algoritmo de Yates do método fatorial foi possível determinar a influência dos parâmetros sobre a resposta experimental. As respostas, R1 e R2, obtidas nos experimentos de velocidade de sedimentação foram os dados utilizados na construção do algoritmo de Yates. Os resultados obtidos com o algoritmo de Yates possibilitaram a otimização da velocidade de sedimentação, quantidade ideal de reagentes e a variação de $\mathrm{pH}$ pelo método do Passo Ascendente. O termo otimização se refere ao método de se definir o melhor caminho para se alcançar uma melhoria do processo, caso haja.

Nessa condição ideal, todas as variáveis importantes devem ser otimizadas, favorecendo a maximização da resposta. Através do Método de Otimização do Passo Ascendente (Duckworth, 
1960; Box et al., 1988), buscou-se determinar as condições ótimas de operação das variáveis de maior influência no processo selecionadas no planejamento fatorial de $2^{3}$. 0 método fornece a projeção da resposta estatística e os valores das demais variáveis são obtidos simultaneamente. As limitações tecnológicas de cada variável além de fornecer os valores máximos que podem ser adotados, aparece também como critério de seleção das condições ótimas do processo.

Experimentos duplicados em bancada de laboratório foram realizados, empregando-se as condições experimentais otimizadas pelo Método do Passo Ascendente. O objetivo foi realizar uma análise comparativa entre os resultados experimentais alcançados nestes testes com aqueles previstos pela metodologia estatística.

\section{MATERIAIS E MÉTODOS}

A amostra utilizada é uma lama proveniente do processamento de minério de fosfato.

Para a caracterização mineralógica foi utilizada a Difração de Raios X: Os difratogramas foram obtidos pelo método do pó, foram coletados em um equipamento Philips-PANalytical, modelo EMPYREAN, nas seguintes condições de operação: radiação CuKa e monocromador $\lambda=1,54184 \AA$; velocidade do goniômetro de $0,06^{\circ}(2 \theta)$ por passo com tempo de contagem de 1 segundos por passo e coletados de 3,03 a 89,97ㅇ (20). As identificações de fases cristalinas por difração de raio $X$ deverá ser analisadas pelo softwares High Score Plus, aonde os picos de cada fase presente no banco de dados são comparados com o difratograma da amostra, atribuindo-se créditos/penalidades para cada pico difratado, no que se refere a presença / ausência de fases, intensidade difratada e deslocamento da distancia interplanar. Ao final do processo de busca e comparação, os resultados são expressos em ordem decrescente de pontuação, seguindo-se a avaliação final por parte do usuário. As interpretações qualitativas dos difratogramas foram efetuadas por comparação com padrões contidos no banco de dados ICSD em software High Score Plus da PANalytical e refinamento de Rietveld com o uso do software GSAS são várias as fases que podem ser quantificadas. e o método de Rietveld foram utilizados na determinação quantitativa da composição mineralógica da amostra.

Reagentes agregantes foram utilizados nos testes de sedimentação: floculantes comerciais (poliacrilamidas) do tipo catiônico (Floticor 7464), aniônico (Floticor 7130), não iônico (Floticor 28755); coagulante sulfato de alumínio.

Os testes de sedimentação foram realizados com polpas contendo $10 \%$ de sólidos em massa e utilizando-se proveta de $2000 \mathrm{~mL}$. Foram realizados primeiramente testes de sedimentação para cada tipo de floculante em várias dosagens. E em seguida foram realizados testes de sedimentação com adição prévia de coagulante para cada dosagem de floculante.

As variáveis estudadas e os valores do nível superior (+) e do nível inferior (-) considerados para os testes estão mostrados nas Tabelas 1,2 e 3.

No planejamento experimental foram realizados ensaios em duplicata para cada condição testada.

Tabela 1: Valores das variáveis estudadas nos testes com floculante catiônico. 


\begin{tabular}{lcc}
\hline Variáveis & $\begin{array}{c}\text { Nível superior } \\
(+)\end{array}$ & Nível inferior (-) \\
\hline A -pH & 12 & 6,5 \\
$\begin{array}{l}\text { B- dosagem de } \\
\text { coagulante (sulfato } \\
\text { de alumínio (g/t)) }\end{array}$ & 99,44 & 27,62 \\
$\begin{array}{l}\text { C- dosagem de } \\
\text { floculante (Floticor }\end{array}$ & 22,10 & \\
$7464(\mathrm{~g} / \mathrm{t})$ ) & & 5,53 \\
\hline
\end{tabular}

Tabela 2: Valores das variáveis estudadas nos testes com floculante aniônico.

\begin{tabular}{lcc}
\hline Variáveis & Nível superior & Nível inferior \\
\hline A pH & 12 & 6,5 \\
$\begin{array}{l}\text { B- dosagem de } \\
\text { coagulante (sulfato } \\
\text { de alumínio (g/t)) }\end{array}$ & 99,44 & 27,62 \\
$\begin{array}{l}\text { C- dosagem de } \\
\text { floculante (Floticor }\end{array}$ & 22,10 & \\
$7130(\mathrm{~g} / \mathrm{t})$ ) & & 5,53 \\
\hline
\end{tabular}

Tabela 3: Valores das variáveis estudadas nos testes com floculante não iônico.

\begin{tabular}{lcc}
\hline Variáveis & Nível superior & Nível inferior \\
\hline A pH & 12 & 6,5 \\
$\begin{array}{l}\text { B- dosagem de } \\
\text { coagulante (sulfato } \\
\text { de alumínio (g/t)) }\end{array}$ & 99,44 & 27,62 \\
$\begin{array}{l}\text { C- dosagem de } \\
\text { floculante (Floticor }\end{array}$ & 44,20 & \\
$28755(\mathrm{~g} / \mathrm{t}))$ & & 5,53 \\
\hline
\end{tabular}

\section{RESULTADOS E DISCUSSÃO}

A difração de raios $X$ e o método de Rietveld indicaram que a composição mineralógica da amostra é $80,57 \%$ calcita magnesiana; $11,10 \%$ dolomita; $6,72 \%$ fluor apatita; $1,6 \%$ biotita magnesiana. Este resultado está em concordância com os valores normalmente obtidos para lamas com estas características. 
As Tabelas 4 e 5 se referem aos parâmetros da célula unitária de acordo com a quantificação pelo método de Rietveld e os dados padrão ICSD para amostras analisada célula unitária $(a, b, c, \alpha, \beta, \gamma)$.

Tabela 4: Os parâmetros da célula unitária de acordo com a quantificação pelo método de Rietveld

\begin{tabular}{|c|c|c|c|c|c|c|}
\hline Fases & $a(\AA ̊)$ & $b(\AA ̊)$ & $c(\AA ̊)$ & $\alpha$ & $\beta$ & $v$ \\
\hline $\begin{array}{l}\text { 1:Biotita magnesiana } \\
(\mathrm{Mn}, \mathrm{Mg}, \mathrm{Al}, \mathrm{Fe}) 1,8(\mathrm{~K}, \mathrm{Na}, \mathrm{Ca}) 0 \\
, 91(\mathrm{Si}, \mathrm{Al}, \mathrm{Ti}) 4(\mathrm{O}, \mathrm{OH}) 12\end{array}$ & 5,358225 & 9,374507 & 10,308416 & 90,000 & 100,437 & 90,000 \\
\hline $\begin{array}{l}\text { 2:Calcita magnesiana } \\
(\mathrm{Mg} 0,03 \mathrm{CaO}, 97)(\mathrm{CO} 3)\end{array}$ & 4,979839 & 4,979839 & 17,022907 & 90,000 & 90,000 & 120,000 \\
\hline $\begin{array}{l}\text { 3: Dolomita } \\
\text { CaMg0,77Fe0,23(CO3)2 }\end{array}$ & 4,816433 & 4,816434 & 16,036430 & 90,000 & 90,000 & 120,000 \\
\hline $\begin{array}{l}\text { 4: Fluor Apatita } \\
\text { Ca5(PO4)3F }\end{array}$ & 9,399364 & 9,399364 & 6,888951 & 90,000 & 90,000 & 120,000 \\
\hline
\end{tabular}

Tabela 5 : Dados padrão ICSD para a amostra analisada célula unitária $(a, b, c, \alpha, \beta, \gamma)$

\begin{tabular}{|c|c|c|c|c|c|c|c|c|}
\hline Fases & ICSD & $\begin{array}{l}\text { Grupo } \\
\text { Espacial }\end{array}$ & $\mathrm{a}(\AA ̊)$ & $b(\AA)$ & $c(\AA)$ & $\alpha$ & $\beta$ & $\gamma$ \\
\hline $\begin{array}{l}\text { 1:Biotita magnesiana } \\
(\mathrm{Mn}, \mathrm{Mg}, \mathrm{Al}, \mathrm{Fe}) 1,8(\mathrm{~K}, \mathrm{Na}, \mathrm{Ca} \\
0,91(\mathrm{Si}, \mathrm{Al}, \mathrm{Ti}) 4(\mathrm{O}, \mathrm{OH}) 12\end{array}$ & 880709 & $\mathrm{C} 2 / \mathrm{m}$ & 5,359998 & 9,374866 & 10,309367 & 90,000 & 100,448 & 90,000 \\
\hline $\begin{array}{l}\text { 2: Calcita magnesiana } \\
(\mathrm{Mg} 0,03 \mathrm{CaO}, 97)(\mathrm{CO} 3)\end{array}$ & 891304 & $R-3 c$ & 4,980143 & 4,980143 & 17,022623 & 90,000 & 90,000 & 120,000 \\
\hline $\begin{array}{l}\text { 3: Dolomita } \\
\text { CaMg0,77Fe0,23(CO3)2 }\end{array}$ & 842065 & $\mathrm{R}-3$ & 4,816451 & 4,816451 & 16,036707 & 90,000 & 90,000 & 120,000 \\
\hline $\begin{array}{l}\text { 4: Fluor Apatita } \\
\text { Ca5(PO4)3F }\end{array}$ & 872462 & $3 / \mathrm{m}$ & 9,399364 & 9,399364 & 6,889074 & 90,000 & 90,000 & 120,000 \\
\hline
\end{tabular}

A Figura 7 representa o refinamento (método Rietveld) usando o programa GSAS/EXPGUI com os dados obtidos a partir da análise de difração de raios $X$ da amostra de fluorapatita. 0 refinamento alcançou seguintes valores: $\operatorname{Rwp}=0,1079$ ou $10,79 \%, R p=0,0805$ ou $8,05 \%$ 


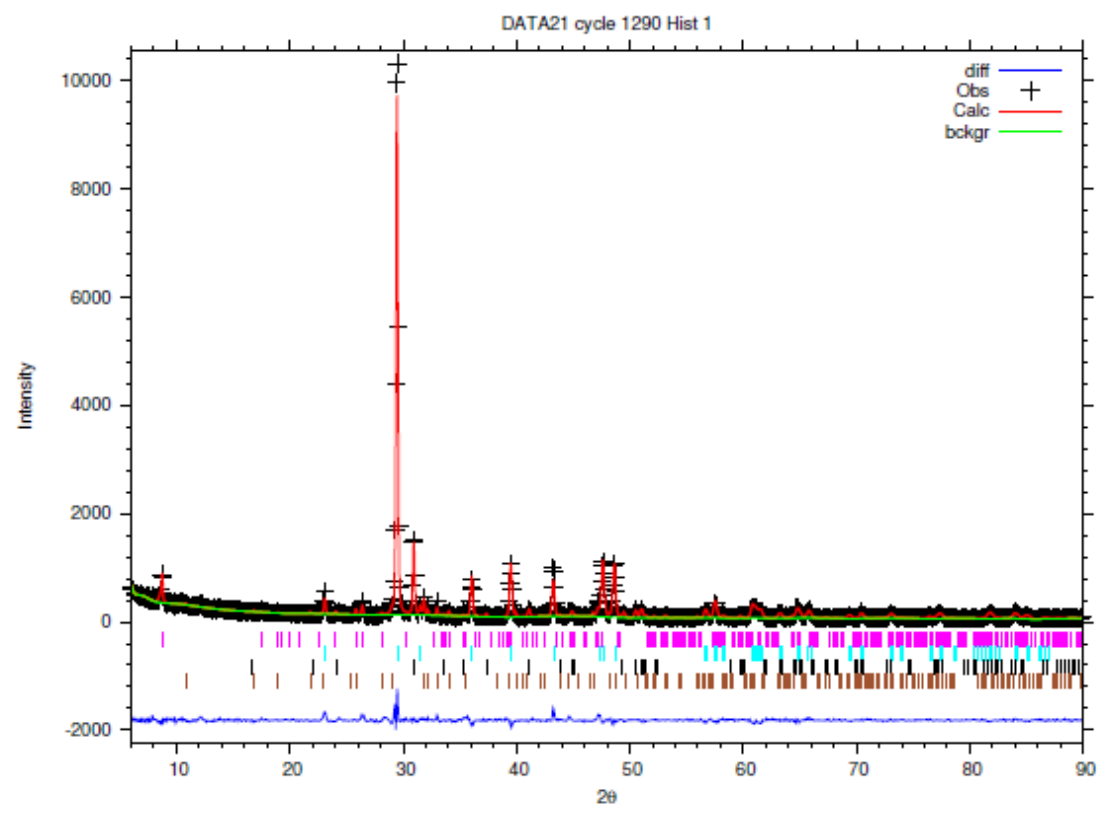

Figura 7: Refinamento (método Rietveld) usando o programa GSAS/EXPGUI com os dados obtidos a partir da análise de difração de raios $X$ da amostra de fluorapatita

A Tabela 6 pode ser observada as densidades de cada fase de acordo com o padrão ICSD com as densidades calculadas pelo Método de Rietveld.

Tabela 6: Comparação entre as densidades de cada fase de acordo com o padrão ICSD com as densidades calculadas pelo Método de Rietveld:

\begin{tabular}{llll}
\hline Fases & $\begin{array}{l}\text { Densidades } \\
(\mathrm{g} / \mathrm{cm} 3)\end{array}$ & padrão ICSD & $\begin{array}{l}\text { Densidade calculada } \\
\text { Rietveld }(\mathrm{g} / \mathrm{cm} 3)\end{array}$ \\
\hline $\begin{array}{l}\text { 1: Biotita magnesiana } \\
\text { (Mn,Mg,Al,Fe)1,8(K,Na,Ca)0,91(Si,Al,Ti)4(O,OH)12 }\end{array}$ & 2,877 & 2,709 \\
$\begin{array}{l}\text { 2: Calcita magnesiana } \\
\text { (Mg0,03Ca0,97)(CO3) }\end{array}$ & 2,722 & 2,684 \\
$\begin{array}{l}\text { 3: Dolomita } \\
\text { CaMg0,77Fe0,23(CO3)2 }\end{array}$ & 2,968 & 2,967 \\
$\begin{array}{l}\text { 4: Fluor Apatita } \\
\text { Ca5(PO4)3F }\end{array}$ & 3,205 & 3,177 \\
\hline
\end{tabular}

O planejamento experimental e os resultados obtidos nos testes em duplicata (R1 e R2), em presença do floculante catiônico, estão mostrados na Tabela 7. 
Tabela 7: Planejamento experimental e resultados obtidos nos testes em duplicata (R1 e R2), em presença do floculante catiônico.

\begin{tabular}{lllllll}
\hline Experimentos & Notação & \multicolumn{2}{l}{ Variáveis } & \multicolumn{3}{c}{ Respostas (cm/s) } \\
\cline { 3 - 7 } & & A & B & C & R1 & R2 \\
1 & i & - & - & - & 0,046 & 0,051 \\
2 & a & + & - & - & 0,058 & 0,037 \\
3 & b & - & + & - & 0,025 & 0,027 \\
4 & ab & + & + & - & 0,032 & 0,044 \\
5 & c & - & - & + & 0,419 & 0,413 \\
6 & ac & + & - & + & 0,178 & 0,167 \\
7 & bc & - & + & + & 0,171 & 0,169 \\
8 & abc & + & + & + & 0,103 & 0,127 \\
\hline
\end{tabular}

A Tabela 8 mostra o resultado do Teste de Fisher para a validade do Método de Yates para o floculante catiônico.

Tabela 8: Aplicação do Teste de Fisher para a validade do Método de Yates.

\begin{tabular}{lllllllll}
\hline Código & Coeficientes & $\mathrm{xA}$ & $\mathrm{xB}$ & $\mathrm{xC}$ & $\mathrm{R}$ & $\mathrm{SQR}$ & $\mathrm{R} 1$ & $\mathrm{R} 2$ \\
\hline 1 & 0,129 & -1 & -1 & -1 & 0,048 & $1,3^{*} 10^{-5}$ & 0,046 & 0,051 \\
$\mathrm{a}$ & $-0,036$ & +1 & -1 & -1 & 0,048 & $-2,2^{*} 10^{-4}$ & 0,058 & 0,037 \\
$\mathrm{~b}$ & $-0,042$ & -1 & +1 & -1 & 0,026 & $-2,0 * 10^{-6}$ & 0,025 & 0,027 \\
$\mathrm{ab}$ & 0,025 & +1 & +1 & -1 & 0,038 & $7,2^{*} 10^{-5}$ & 0,032 & 0,044 \\
$\mathrm{C}$ & 0,089 & -1 & -1 & +1 & 0,416 & $1,8^{*} 10^{-5}$ & 0,419 & 0,413 \\
$\mathrm{ac}$ & $-0,039$ & +1 & -1 & +1 & 0,172 & $-6,1^{*} 10^{-5}$ & 0,178 & 0,167 \\
$\mathrm{bc}$ & $-0,034$ & -1 & +1 & +1 & 0,170 & $-2,0^{*} 10^{-6}$ & 0,171 & 0,169 \\
$\mathrm{abc}$ & 0,022 & +1 & +1 & +1 & 0,114 & $2,9^{*} 10^{-4}$ & 0,103 & 0,127 \\
\hline
\end{tabular}

Os valores previstos pelo modelo $(R)$ em função dos valores experimentais são observados através da Figura 8. Verifica-se que o ajuste obtido é muito bom.

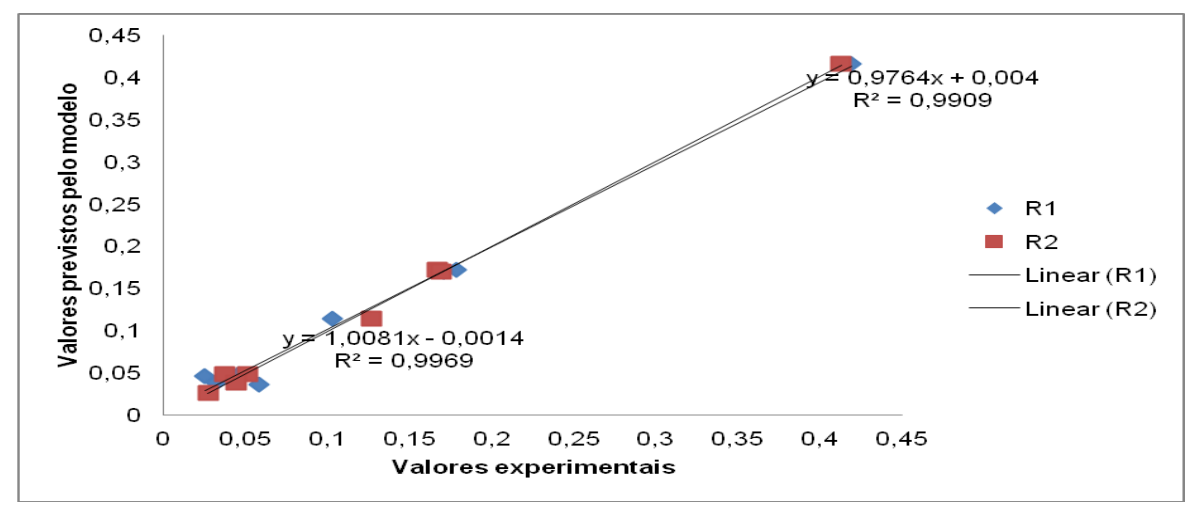

Figura 8: Valores previstos pelo modelo (R) em função dos valores experimentais.

A equação 1 obtida considerando-se o Teste de Fisher é: 
$R=0,129-0,036 \times A-0,042 \times B+0,025 \times A B+0,089 \times C-0,039 \times A C-0,034 \times B C+0,022 \times A B C$.

Observe-se que a variável de maior influência é a dosagem de floculante, pois quando se passa do nível inferior para o nível superior a influencia é positiva. A segunda variável de maior influencia é a dosagem de coagulante, porém quando se passa do nível inferior para o nível superior tem influência negativa.

O planejamento experimental e os resultados obtidos nos testes em duplicata (R1 e R2), em presença do floculante aniônico, estão mostrados na Tabela 9.

Tabela 9: Planejamento experimental e resultados obtidos nos testes em duplicata (R1 e R2), em presença do floculante aniônico.

\begin{tabular}{|c|c|c|c|c|c|c|}
\hline \multirow[t]{2}{*}{ Experimentos } & \multirow[t]{2}{*}{ Notação } & \multicolumn{3}{|c|}{ Variáveis } & \multicolumn{2}{|c|}{ Respostas (cm/s) } \\
\hline & & $A$ & $B$ & $\mathrm{C}$ & R1 & $\mathrm{R} 2$ \\
\hline 1 & $\mathrm{i}$ & - & - & - & 0,043 & 0,042 \\
\hline 2 & $a$ & + & - & - & 0,079 & 0,095 \\
\hline 3 & b & - & + & - & 0,038 & 0,045 \\
\hline 4 & $a b$ & + & + & - & 0,049 & 0,040 \\
\hline 5 & c & - & - & + & 0,160 & 0,138 \\
\hline 6 & ac & + & - & + & 0,199 & 0,192 \\
\hline 7 & bc & - & + & + & 0,159 & 0,149 \\
\hline 8 & $a b c$ & + & + & + & 0,378 & 0,357 \\
\hline
\end{tabular}

A Tabela 10 mostra o resultado do Teste de Fisher para a validade do Método de Yates para o floculante aniônico.

Tabela 10: Aplicação do Teste de Fisher para a validade do Método de Yates.

\begin{tabular}{lllllllll}
\hline Código & Coeficientes & $\mathrm{xA}$ & $\mathrm{xB}$ & $\mathrm{xC}$ & $\mathrm{R}$ & $\mathrm{SQR}$ & $\mathrm{R} 1$ & $\mathrm{R} 2$ \\
\hline 1 & 0,1350 & -1 & -1 & -1 & 0,042 & $1,0^{*} 10^{-6}$ & 0,043 & 0,042 \\
$\mathrm{a}$ & 0,0420 & +1 & -1 & -1 & 0,087 & $1,3 * 10^{-4}$ & 0,079 & 0,095 \\
$\mathrm{~b}$ & 0,0165 & -1 & +1 & -1 & 0,030 & $2,9 * 10^{-4}$ & 0,038 & 0,045 \\
$\mathrm{ab}$ & 0,0190 & +1 & +1 & -1 & 0,058 & $4,1 * 10^{-5}$ & 0,049 & 0,040 \\
$\mathrm{c}$ & 0,0815 & -1 & -1 & +1 & 0,149 & $2,4 * 10^{-4}$ & 0,160 & 0,138 \\
$\mathrm{ac}$ & 0,0230 & +1 & -1 & +1 & 0,196 & $2,5^{*} 10^{-5}$ & 0,199 & 0,192 \\
$\mathrm{bc}$ & 0,0275 & -1 & +1 & +1 & 0,087 & $9,0 * 10^{-3}$ & 0,159 & 0,149 \\
$\mathrm{abc}$ & 0,0225 & +1 & +1 & +1 & 0,367 & $2,2^{*} 10^{-4}$ & 0,378 & 0,357 \\
\hline
\end{tabular}

Os valores previstos pelo modelo $(R)$ em função dos valores experimentais são observados através da Figura 9. Verifica-se que o ajuste obtido é muito bom, mas menor do que aquele obtido para o floculante aniônico. 


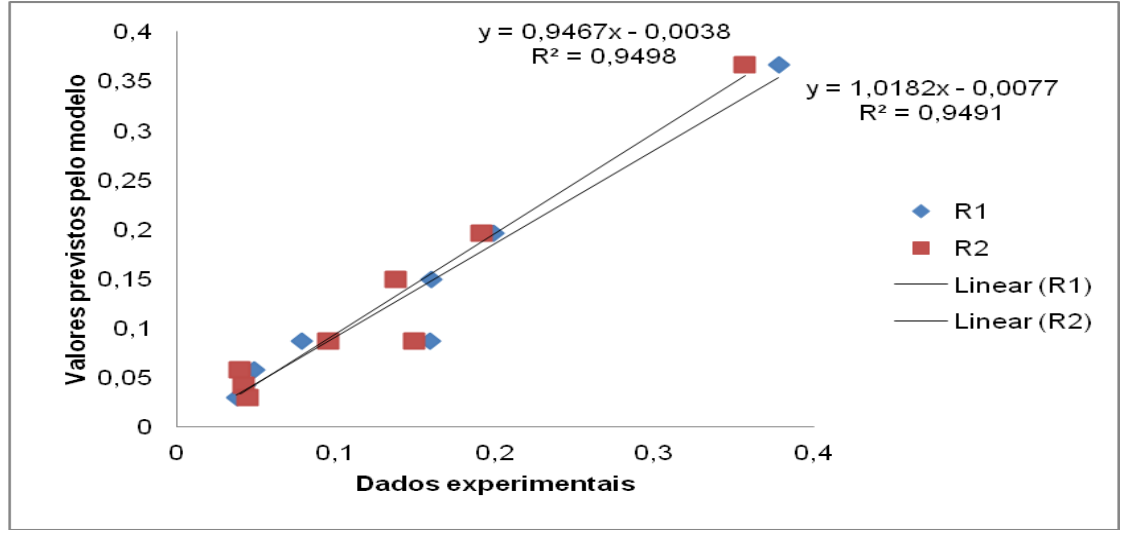

Figura 9: Valores previstos pelo modelo (R) em função dos valores experimentais.

A equação 2 obtida considerando-se o Teste de Fisher é:

$R=0,1350+0,0420 x A+0,0165 x B+0,0190 x A B+0,08150 x C+0,0230 x A C+0,0275 \times B C+0,0225$ $\mathrm{XABC}$

Verifica-se que a variável de maior influência é a dosagem de floculante seguida pelo $\mathrm{pH}$, pois quando se passa do nível inferior para o nível superior a influência é positiva em ambas variáveis.

O planejamento experimental e os resultados obtidos nos testes em duplicata (R1 e R2), em presença do floculante aniônico, estão mostrados na Tabela 11.

Tabela 11: Planejamento experimental e resultados obtidos nos testes em duplicata (R1 e R2), em presença do floculante não iônico.

\begin{tabular}{lllllll}
\hline Experimentos & Notação & \multicolumn{2}{l}{ Variáveis } & \multicolumn{3}{l}{ Respostas (cm/s) } \\
\cline { 3 - 7 } & & A & B & C & R1 & R2 \\
1 & i & - & - & - & 0,005 & 0,068 \\
2 & a & + & - & - & 0,113 & 0,199 \\
3 & b & - & + & - & 0,050 & 0,056 \\
4 & ab & + & + & - & 0,141 & 0,137 \\
5 & c & - & - & + & 0,138 & 0,132 \\
6 & ac & + & - & + & 0,433 & 0,317 \\
7 & bc & - & + & + & 0,130 & 0,155 \\
8 & abc & + & + & + & 0,436 & 0,304 \\
\hline
\end{tabular}

A Tabela 12 mostra o resultado do Teste de Fisher para a validade do Método de Yates para a poliacrilamida não iônica. 
Tabela 12: Aplicação do Teste de Fisher para a validade do Método de Yates.

\begin{tabular}{lllllllll}
\hline Código & Coeficientes & $\mathrm{XA}$ & $\mathrm{xB}$ & $\mathrm{xC}$ & $\mathrm{R}$ & $\mathrm{SQR}$ & $\mathrm{R} 1$ & $\mathrm{R} 2$ \\
\hline 1 & 0,178 & -1 & -1 & -1 & 0,0278 & 0,0021 & 0,005 & 0,068 \\
$\mathrm{a}$ & 0,118 & +1 & -1 & -1 & 0,2273 & 0,0138 & 0,113 & 0,199 \\
$\mathrm{~b}$ & $2,500 * 10^{-4}$ & -1 & +1 & -1 & 0,0193 & 0,0023 & 0,050 & 0,056 \\
$\mathrm{ab}$ & $-0,008$ & +1 & +1 & -1 & 0,2647 & 0,0316 & 0,141 & 0,137 \\
$\mathrm{C}$ & 0,080 & -1 & -1 & +1 & 0,1273 & 0,0001 & 0,138 & 0,132 \\
$\mathrm{ac}$ & $-0,001$ & +1 & -1 & +1 & 0,3762 & 0,0037 & 0,433 & 0,317 \\
$\mathrm{bc}$ & 0,004 & -1 & +1 & +1 & 0,1507 & 0,0005 & 0,130 & 0,155 \\
$\mathrm{abc}$ & $5,630 * 10^{-4}$ & +1 & +1 & +1 & 0,3698 & 0,0087 & 0,436 & 0,304 \\
\hline
\end{tabular}

Os valores previstos pelo modelo $(\mathrm{R})$ em função dos valores experimentais são observados através da Figura 10. Verifica-se que o ajuste obtido é menor do que aqueles obtidos para os floculantes catiônico e aniônico.

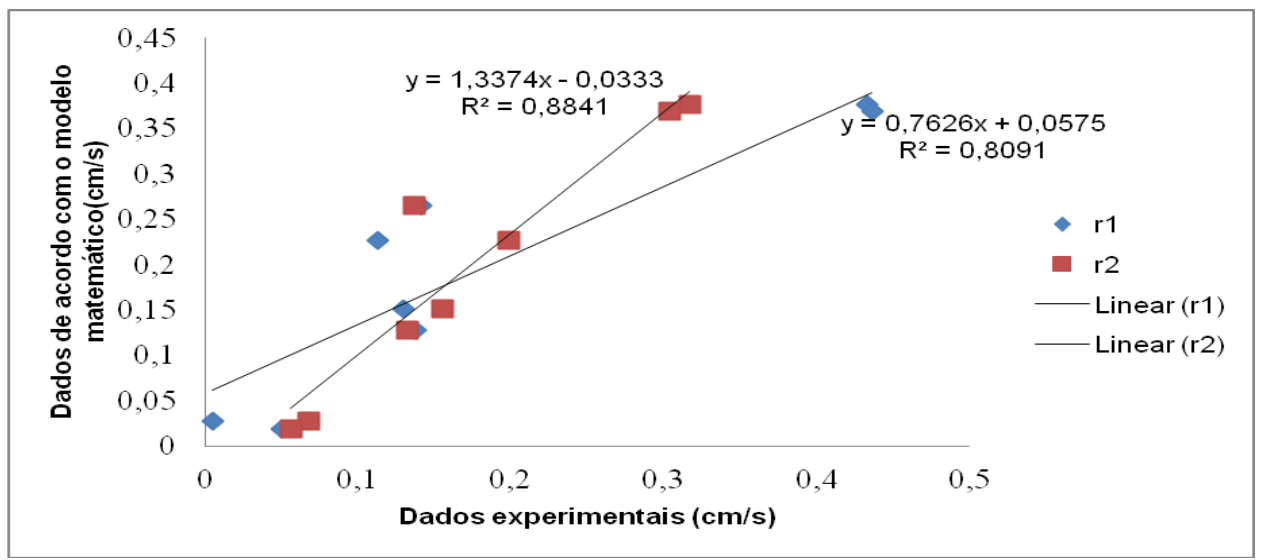

Figura 10: Valores previstos pelo modelo $(R)$ em função dos valores experimentais.

A equação 3 obtida considerando-se o Teste de Fisher é:

$R=0,176+0,118 \times A+2,50 * 10^{-4} \times B-0,008 \times A B+0,080 \times C-0,001 \times A C+0,004 \times B C+5,63 * 10^{-4} \times A B C$

Note-se que a variável de maior influência é o pH seguida da dosagem de coagulante, pois quando se passa do nível inferior para o nível superior a influência é positiva.

A otimização dos resultados pelo Método do Passo Ascendente, tanto para o floculante catiônico quanto para o floculante aniônico, indicou que as melhores condições de sedimentação são obtidas para: pH 9,25, dosagem de coagulante 63,54g/t e dosagem de floculante 13,81g/t.

Com esta otimização a maior velocidade de sedimentação é de $2,62 \times 10^{-1} \mathrm{~cm} / \mathrm{s}$.

Quando é realizada a otimização dos resultados pelo Método do Passo Ascendente para o floculante não iônico obteve-se: $\mathrm{pH}=9,57$, dosagem de coagulante $63,54 \mathrm{~g} / \mathrm{t}$ e dosagem de floculante $22,04 \mathrm{~g} / \mathrm{t}$.Com esta otimização a maior velocidade de sedimentação é de $1,09 \times 10^{-1} \mathrm{~cm} / \mathrm{s}$. 


\section{CONCLUSÕES}

Na composição mineral predomina calcita-Mg (80\%), em menores quantidades dolomita, fluor apatita e biotita.

- $\quad$ Nos testes realizados, dois variáveis influenciam a velocidade de sedimentação, a dosagem de reagentes (floculante catiônico e aniônico) e o pH (floculante não iônico). Dentre das variáveis de maior influência é a poliacrilamida de carga catiônica.

A otimização dos resultados, pelo Método do Passo Ascendente, indicou tanto para o floculante catiônico quanto para o floculante aniônico, que as melhores condições de sedimentação são obtidas para: pH 9,25, dosagem de coagulante 63,54g/t e dosagem de floculante $13,81 \mathrm{~g} / \mathrm{t}$. Com esta otimização a maior velocidade de sedimentação é de $2,62 \times 10^{-1} \mathrm{~cm} / \mathrm{s}$. Utilizando-se este método para o floculante não iônico obteve-se: $\mathrm{pH}=9,57$, dosagem de coagulante $63,54 \mathrm{~g} / \mathrm{t}$ e dosagem de floculante $22,04 \mathrm{~g} / \mathrm{t}$. Com esta otimização a maior velocidade de sedimentação é de $1,09 \times 10^{-1} \mathrm{~cm} / \mathrm{s}$.

\section{REFERÊNCIAS}

BALTAR, C.A.M.Processos de Agregação. In: BENVINDO, A.L et al. Tratamento de Minérios. 5a Edição. CETEM - Centro de Tecnologia Mineral; Rio de Janeiro-Brasil, p.559-594,2010.

BOX , G.P.; HUNTER , W.G.; HUNTER , P.J. 1988. Statistics for experimenters. New York, Ed. John Wiley \& Sons Inc., 653 p.

CHAVES, A.P. e colaboradores, Teoria e Prática do Tratamento de Minérios, Vol. II, Desaguamento, Espessamento e Filtragem. Brasil - São Paulo, 2004.

CLAESSON, P.M., DAHLGREN, M.A.G., ERIKSSON, L., 1994. Forces between Polyelectrolyte-Coated Surfaces: Relations between Surface Interaction and Floc Properties, Colloids and Surfaces, vol. 93, pp. 293-303

DUCKWORTH, W.E. 1960. Statistical techniques in technological research. Londres, Ed. Methuen \& Co. Ltd.,303 p.

FAUST, A. S. (1982). Princípios das Operações Unitárias. Rio de Janeiro: LTC.

HOGG, R. Flocculation Dewatering. International Journal of Mineral Processing, 58 (1-4), 223-236, 2000.

HUNTER, R. J. Introduction to modern colloid science. 1a Ed. Oxford: Oxford University Press, 1994. 352p. (Oxford Science Publications

KIM, Y. H. Coagulants and flocculants; theory and practice, 1a Ed. Littleton: Tall Oaks Publishing, Inc., 1995. 85p.

KIRWAN, L. J. An Investigation of polyacrylate adsorption onto hematite. Perth: Curtin University of Technology, 2002, 191p. (Tese, Doutorado em Química Aplicada).

KISSA, E. Dispersions; characterization, testing and measurement. 1a Ed. New York: Marcel Dekker, Inc., 1999. 724p. (Surfactant Science Series, vol. 84). 
LA MER, V. K. \& HEALY, T. W. The role of filtration in investigating flocculation and redispersion of colloidal dispersions. Journal of Physical Chemistry, vol. 67, no 11, pp.2417-2420, novembro, 1963

LUZ, A. B., SAMPAIO, J. A. e ALMEIDA, S. L. M.. Tratamento de Minérios. 4ạ Ed. Rio de Janeiro: CETEM/MCT, dezembro, 2004. 867p. Cap. 14.

LUZ, A. B. et al. (2002). Tratamento de Minérios. 3a Edição. CETEM - Centro de Tecnologia Mineral; Rio de Janeiro-Brasil.

MONTGOMERY, P. C.; RUNGER, G. C. Estatística Aplicada e Probabilidade para Engenheiros. 2 ed. Rio de Janeiro: LTC, 2003

RICHSTFDON, P.F.; CONNELLY, L.J. Industrial Coagulants and Flocculants. In.: Reagents in Mineral Technology. Somasundaran, P. and Moudgil, B.M. (editors). Marcel Dekker, Inc., 519$558,1988$.

SHARMA, B.R., DHULDHOYA, N.C., MERCHANT, U.C. (2006). Flocculants Ecofriendly Approach. Journal Polymer Environmental, 14, 195- 202

SWIFT, J. D., SIMIC, K., JOHNSTON, R. R. M., FAWELL, P. D. e FARROW, J. B. (2004). A study of the polymer flocculation reaction in a linear pipe with a focused beam reflectance measurement probe. International Journal of Mineral Processing,vol. 73, no 2-4, pp.103-118

VALADÃO, G.E. (2011). Notas de Aula do Curso de Especialização em Recursos Minerais - DEMIN: ERM 526 - Separação Sólido- Liquido I. Belo Horizonte, Brasil: Universidade Federal de Minas Gerais

VERRAL K., WARWICK, P. e FAIRHURST, A.(1999). Application of Schulze-Hardy rule to haematite and haematite/humate colloid stability. Colloids and Surfaces A:Phisicochemical and Engineering Aspects, vol. 150, pp.261-273 